\title{
Automation in Blur Image Detection with Segmentation using Machine learning
}

\author{
Mahak Gupta ${ }^{1}$, Jaspreet Kaur ${ }^{2}$ \\ Research Scholar, ECE, Rayat Bahra Univeristy, Mohali, India ${ }^{1}$ \\ Assistant Professor, ECE, Rayat Bahra Univeristy, Mohali, India ${ }^{2}$
}

\begin{abstract}
One of the definition of blurring with respect to Laplacian is decrease in the variance. Blurring with respect to frequency domain means low frequency where high frequency represents edge. It occurs either because of motion or out of focus parameter. Blur is classified in two types (Local and Global Blur). In this research paper, both the blurred and unblurred images are passed through Fourier transform which calculates the high frequencies as well as low frequencies from the images. In other words, it transforms image from spatial domain to frequency domain. Labels for both blurred as well as unblurred images are chosen as the target i.e. 1 for blurred and 0 for unblurred. These set of images are trained using both support vector machine as well as logistic regression which are tested on the real time images which detects the blur from the images. Trained Model run over the real time image and captures the blur from it. Both state of art method i.e. support vector machine as well as logistic regression is compared in the terms of performance parameters and it is concluded from the results that later winds over the former in both accuracy as well as receiver operating characteristic curve.
\end{abstract}

Keywords: Support vector machine, Logistic regression, binary values, receiver operating characteristic curve

\section{INTRODUCTION}

Blurring is a concerning issue [1]. An image looks clear due to its edges. Blurring in simple sense means reducing the edges. In technical language, blurred image is combination of sharp image and point spread function. The shape of PSF (Point Spread Function) depends on type of blur. If it (blur) is due to out of focus, PSF will be a circle. If it is due to motion, PSF will be a line or curve. Mathematically blurring is defined as convolutional of sharp image and point spread spectrum.

$$
\begin{aligned}
\mathrm{f}(\mathrm{t}) & =\text { input image } \\
\mathrm{g}(\mathrm{t}) & =\text { point spread function } \\
\mathrm{f}(\mathrm{t}) * \mathrm{~g}(\mathrm{t}) & =\int_{-\infty}^{\infty} \mathrm{f}(\tau) \mathrm{g}(\mathrm{t}-\tau) \mathrm{d} \tau
\end{aligned}
$$

Blurring in image is of two types. Local and global blur. Local blur refers to a blur where some portion of image is degraded while other is clear at the same time while in global blurring image is blurred throughout. Once the blur is detected, it is extracted using specially designed blur features such as gradient or color information. A neural network based approach [2] for finding type of blur was proposed by I. Aizenberg. He used two single layer multivalued neural network. Since input is discrete, algorithm suffers from quantization error which was removed with the use of continuous values. Ali and Mahmood [3] has proposed IQR based blur classification in which they have used initial blur, normalization and IQR for blur segmentation as well as classification. There is another way of distinguish blur image from unblur image. It is by calculating the variance of the laplacian of an image. Laplacian refers to calculating the second order derivative of an image. It highlights the region whose intensity value changes rapidly. High variance represents wide spread of response which is an edge whereas low variance represents tiny spread of the response which signifies blur. Let $\mathrm{x}$ be the laplacian random variable whose variance is to be calculated.

$$
\begin{aligned}
& \text { Probability density function }(P D F)=f_{X}(x)=\frac{\partial}{2} \\
& \quad-\partial<x<\partial \\
& \text { Expectation of } x=E(x)=\int_{-\infty}^{\infty} \mathrm{xf}_{\mathrm{X}}(\mathrm{x}) \mathrm{dx} \\
& \text { Variance }[\mathrm{x}]=\mathrm{E}\left[\mathrm{x}^{2}\right]-\left(\mathrm{E}[\mathrm{x}]^{2}\right)
\end{aligned}
$$


Vol. 8, Issue 3, March 2019

In recent time, Machine learning and artificial intelligence has been quite active and is widely used for automation. One of the reason is high accuracy achieved with respect to traditional methods and second is machine learning works on the principle of pattern matching which is missing in the traditional methods. In today's time, most of the work of computer vision and image processing has been implemented via machine learning such as denoising of image, edge detection, image restoration, image deblurring and many others. This paper proposes an algorithm to detect blur from the image using support vector machine and logistic regression. Model is trained with both blurred and unblurred images. A set of $\mathbf{2 0 0 0}$ images are taken where 1000 are blurred images (500 motion and 500 out of focus) and 1000 were without blur images. Fourier transforms are used to extract the features which will be explained in this introduction part. Once the features are extracted from the images, labels are assigned to the images i.e. 1 for blurred and 0 for unblurred. Only two classes has been takes as we have chosen classification supervised model (support vector machine and logistic regression) for training which binary classifiers are. Please have a look at the below Fig. 1 for training phase.

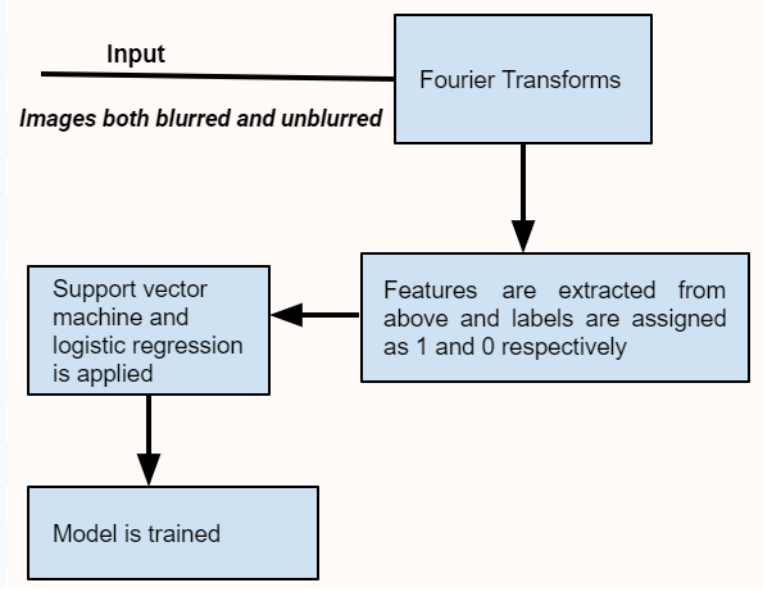

Fig. 1 Training of Model

If carefully examination of the model is done it is concluded that model is trained to predict the blurred images from the unblurred images. Now aim is to detect the blur from the image and segment it. Novelty lies in the fact that blurred images are detected automatically from the list of images passed during testing phase. Please have a look at the testing phase shown in Fig.2.

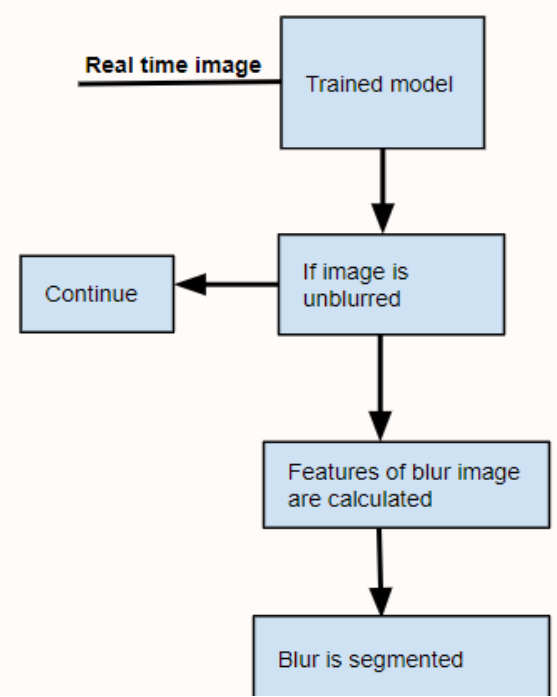

Fig. 2 Prediction Phase

In prediction phase, image is passed to the trained model. Image are applied in a loop. If image detected is unblurred, loop is continued. If image so detected is blurred, it is passed to the feature extraction phase developed during the training phase. Such features are segmented from the image which is the detected blur. Code is implemented with proper coding style and functions. Both Roadmap (Training and Testing) is implemented using Matlab coding. Remaining paper has been organized as follows: Section 2 deals with related work, Section 3 deals with proposed algorithm with equation, Section 4 deals with Experimental results and Simulations and Section 5 with conclusion. 
Vol. 8, Issue 3, March 2019

Before going there will be some general discussion on three techniques used which are Fourier transforms, support vector machines and logistic regressions.

\section{A. Fourier Transforms}

Fourier transforms convert both periodic and aperiodic signals into frequency domain. There is a limitation for Fourier transforms that they act only on stable signals only. It is the advanced version of Fourier series which is applicable for only periodic signals. Before going further let us see the mathematically expression for it.

Let $x(t)$ be the signal in the frequency domain. $X(W)=\int_{-\infty}^{\infty} x(t) e^{-j w t} d t$

One of the question that arises here why Fourier transforms is needed and what is the significance of it with respect to blur detection. Let us explain these points with some theory as well as images. The need arises when you are dealing with mixing of different signals in a single signal. You cannot calculate both the frequencies and respective amplitude in time domain. Please have a look at the below Fig. 3

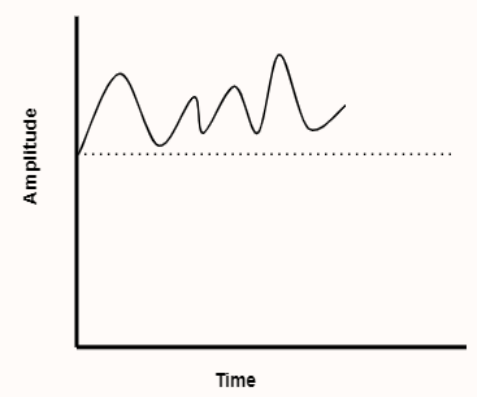

Fig. 3 Signal in time domain

If you analyse the same signal in the frequency domain, you will get something like below as shown in the Fig. 4

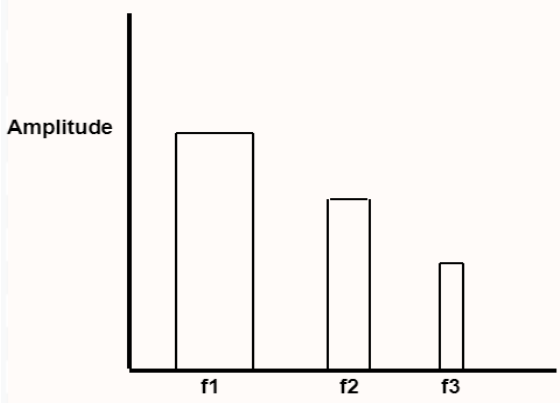

Fig. 4 Signal in frequency domain

This concept is taken into account for image blur detection also. You cannot get much information when you analyse the image in the time domain i.e. spatial domain but when you convert the image into the frequency domain, you will get more pattern analysis as well frequencies image contains. Unblurred images contains high frequencies as it has sharp edges. Edges represents high frequencies whereas blur means absence of blur or lower variance which means presence of lower frequency or generally the absence of high frequency. Using this as an advantage features are extracted for both the types of images which will be fed to the supervised classification model for training the model.

B. Support vector Machines

This paper proposes segmenting blur from the images using machine learning. So before proceeding further, i.e. to discuss about support vector machine or logistic regression, it is important to know about different steps for machine learning. Have a look at the below Fig. 5 for this

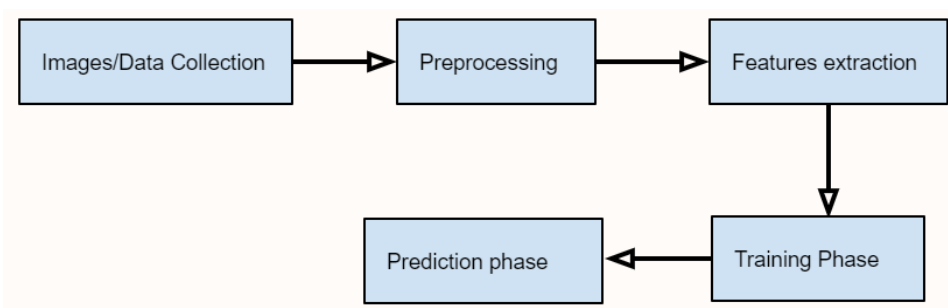

Fig. 5 Machine learning general steps 
Phase 1: Data Collection: This is the first phase and most crucial phase for machine learning. For example collection of blurred and unblurred images is implemented as the first step.

Phase 2: Preprocessing is the second phase where data is checked again and cleaning is done if any abnormalities are confirmed at this stage. It is important to feed the cleaned data to the machine. For example, in this research paper there may be many image which are not easily visible such images are cleaned.

Phase 3: Feature extraction: You cannot feed simply data/images for the model to get trained. It needs features to learn the pattern from the data to perform well on the real time data. To accomplish this task, this step is taken into account.

Phase 4: Training phase: Once the data is prepared, it is fed to the model to get trained. Model selection will depend on type of data.

Phase 5: Once the model is trained, it is ready for the prediction phase. This makes the task automated.

Support vector machines are binary classifier which can be used for multiclass classification using one vs rest approach. Classification problems are the subparts of supervised learning where machines are made to learn with the specific targets [4]. Have a look at the below Fig. 6 to look at how SVM operated and then some points are written for it.

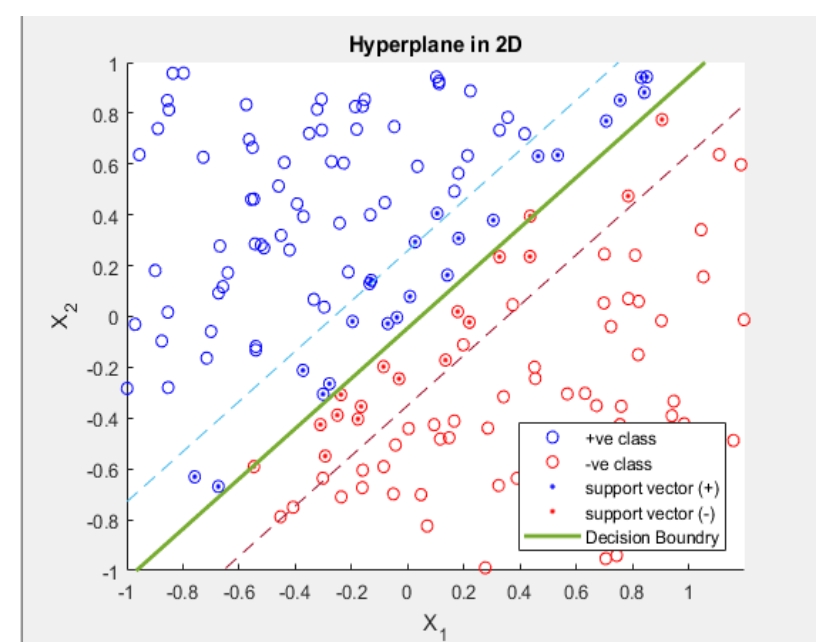

Fig. 6 Support Vector Machine and Hyperplane

1.) Distance between support vectors and the Hyperplane should be as far as possible.

2.) If one is unable to classify the data in the lower plane, plotting is done in the higher plane by adding more features to the data.

3.) SVM works well on the smaller dataset. When dataset is increased, SVM is not preferred. Also it is less effective with the noisy dataset.

4.) It is a very effective technique as it uses subset of training points.

\section{Logistic Regression}

Logistic regression is used to analyse and categorize the target when it is binary. People think of it as a regression model but it is a classification model [5]. In this research paper, it is used to train for blur and deblur images. It can be used for a binary dependent variable to another independent variable.

\section{There are three types of Logistic Regression}

1.) Binary Logistic Regression: It has two possible outcomes or resultants.

2.) Multinomial Logistic Regression: It has three or more categories for an input.

3.) Ordinary Logistic Regression: It has three or more categories with some ordinal choices to predict.

Logistic regression is based on a sigmoid function to describe the properties of an input. It is a monotonic and has a first derivative which is bell shaped [6]. Input values are combined with coefficients/weights to predict the output. It makes prediction based on probability. 0 means total failure, 1 means total success and if the value if between 0 and 1 , it defines the probability of success or failure. In other words, a threshold is selected and if the value is greater than threshold, it denotes success and value below threshold denotes failure. Have a look at the Fig. 7 for understanding Logistic Regression. 
Vol. 8, Issue 3, March 2019

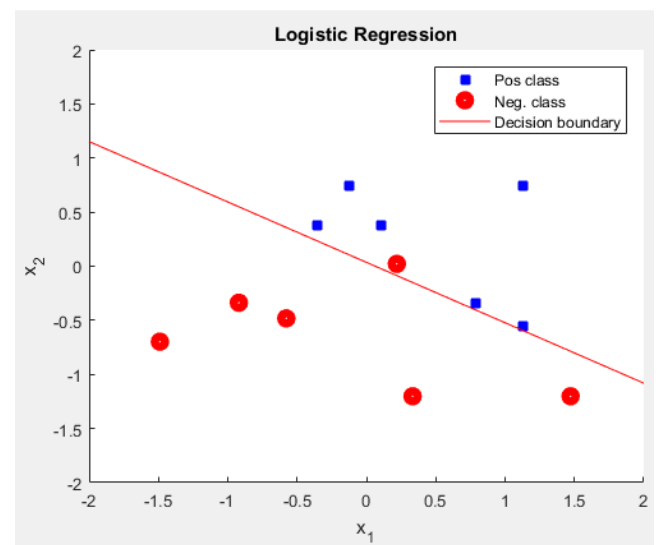

Fig.7 Logistic Regression with decision boundary

II.

\section{RELATED WORK}

Wei et al. [7] worked on Gaussian different signatures to measure the diffuseness of out of focus in the digital images. Such works finds its application in blur estimation based on image intensity.

James et al. [8] worked with blur estimation without labelling image region into blurred and unblurred region. Local scale control is shown to be important for estimation of blur in complex images.

Rob et al. [9] used blind de-convolutional methods in their work with the aim to remove the camera shock from the photograph. Estimation of the blur filter as well as finding the unblurred image is the main focus of this work. Blind refers to the use of the point spread function while reconstructing the normal image from the blind image. One of the advantage for such is detection of potential blur region effectively.

Chen et al. [10] worked on lowest directional high frequency energy. In the previous work [9] point spread function is used for blind de-convolution. This method is a substitute to it and offers a low cost in terms of computation. Blur is detecting by analyzing high frequency energy and is considered as one of the accurate method for blur detection.

Kanchev et al. [11] worked on wavelets to calculate the gradient histograms. These features are subjected to the support vector machine which generates global probability map. From the results and simulation, it is concluded that this method is very effective when the blur is out of focus not on motion blur.

\section{PROPOSED ALGORITHM}

In this paper, a robust algorithm for blur detection and segmentation is proposed. Method is based on Fourier series for blur detection and segmentation, SVM and logistic regression for classification. A set of 2000 images (1000 blur and 1000 unblur) are taken for training purpose. At prediction phase, images are fed to the trained model. If the detected image comes out to be good or unblur image, the loop is continued and next image is looked for it. When blur image is detected by the model, it is passed to the feature extractor phase where blur segmentation takes place. Following section will deal with feature extraction, model training and prediction phase.

A. Feature Extraction using Fourier transforms

Fourier transforms is used to perform correlation which is used to locate features from an image. By taking Fourier transform of an image, frequency analysis of image is implemented. Low frequency data points corresponding to blur features while high frequency data points corresponds to edge features. The following equation is used for calculating the Fourier transform of an image.

$$
X(W)=\int_{-\infty}^{\infty} x(t) e^{-j w t} d t
$$

B. Model training

1.) Support vector machine: Since data is prepared in the Part A, labels are assigned according to the data. Label 1 is assigned for all the images belonging to blur one and label 0 is assigned for all the images belonging to unblur. Data (Features) as well as labels (targets) are passed to the support vector machine with the kernel function as linear. Following equation is used to learn the dataset 
Vol. 8, Issue 3, March 2019

$$
\min _{w \in R^{d}}\|w\|^{2}+C \sum_{I}^{N} \max \left(0,1-y_{i} f\left(x_{i}\right)\right.
$$

This equation is called as objective function while the first term is called as regularization and second is called as loss function. $\mathrm{w}$ is the distance between Hyperplane and support vector. It should be as large as possible. SVM uses hinge loss which is defined as $\sum_{\mathrm{I}}^{\mathrm{N}} \max \left(0,1-\mathrm{y}_{\mathrm{i}} \mathrm{f}\left(\mathrm{x}_{\mathrm{i}}\right)\right.$. Once the model gets trained, model is saved for testing phase. This is implemented using the below code save Compact Model(Model, 'name to be saved') Model is saved in form of mat file which can be loaded when needed. It saves the time for training every time when one have to do prediction.

2.) Logistic Regression: From the name, it looks like that it is regression model but it is also a classifier model. Following equation has been used to train the data and labels with this method.

$$
\mathrm{y}=\frac{\mathrm{e}^{\mathrm{b} 0+\mathrm{b} 1 * \mathrm{x}}}{1+\mathrm{e}^{\mathrm{b} 0+\mathrm{b} 1 * \mathrm{x}}}
$$

$\mathrm{y}$ is the output, $\mathrm{b} 0$ is bias, $\mathrm{b} 1$ is the coefficient and $\mathrm{x}$ is the input

\section{Prediction phase}

Once model is build and saved using save Compact Model, prediction phase is the real time performance analysis of the model. A Number of images are stored in the folder and are loaded one by one to the saved model. These images are applied in a loop keeping the condition that if the unblurred image is detected by the trained model, loop is continued else image so detected is passed to the feature extractor phase where blur data points are highlighted which are segmented there. Please have a look at the pseudo code for it

Step1: for image in (folder)

Step2: if image is not blur, continue else go to Step3

Step3: $\mathrm{ft}$ (image) where $\mathrm{ft}$ is the Fourier transform

Step4: Data points are segmented and plotted for showing blur region.

\section{EXPERIMENTAL RESULTS AND SIMULATIONS}

In this research paper, since images are trained with both classifier models (support vector machine and logistic regression), so parameters which are calculated are as accuracy, precision, recall, F1-score. Let us discuss these but before these these terms needs to be understood.

True Positive (TP): Both actual and predicted results are yes.

True Negative (TN): Both actual and predicted results are no.

False Positive (FP): Actual class is no but predicted class is yes.

False Negative (FN): Actual class is yes but predicted class is no.

Accuracy: It is defined as ratio of correctly predicted observation to the overall observations. Let us see the mathematically formulae for it.

$$
\text { Accuracy }=(\mathrm{TP}+\mathrm{TN}) /(\mathrm{TP}+\mathrm{TN}+\mathrm{FP}+\mathrm{FN})
$$

Recall: It is defined as ratio of correctly predicted positive observation to all observation with the actual class as yes.

$$
\text { Recall }=(\mathrm{TP}) /(\mathrm{TP}+\mathrm{FN})
$$

Precision: It is defined as ratio of correctly predicted positive observation to total predicted positive observation

$$
\text { Precision }=(\mathrm{TP}) /(\mathrm{TP}+\mathrm{FP})
$$

F1-Score: It is defined as the average of recall and precision using the below formulae.

$$
\mathrm{F} 1-\mathrm{Score}=(2 * \text { Recall } * \text { Precision }) /(\text { Recall }+ \text { Precision })
$$

Table I Comparsion Between Algorithms

\begin{tabular}{|l|l|l|}
\hline $\begin{array}{l}\text { Performance Evaluation } \\
\text { Parameters }\end{array}$ & $\begin{array}{l}\text { Support vector } \\
\text { machine in \% }\end{array}$ & $\begin{array}{l}\text { Logistic regression } \\
\text { in \% }\end{array}$ \\
\hline Accuracy & 35 & 96 \\
\hline Recall & 34 & 41 \\
\hline Precision & 50 & 55 \\
\hline F1-Score & 40 & 47 \\
\hline
\end{tabular}


Have a look at the below Fig. 8 for better visualization.

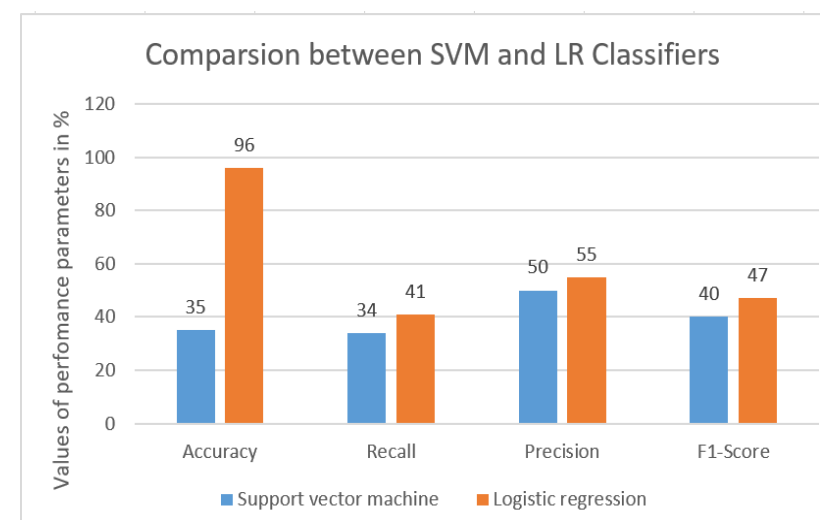

Fig. 8 Comparsion between SVM and LR

\section{CONCLUSION}

In this research paper, literature survey is done on one of hot burning topic under research which is blur detection and segmentation using machine learning. Machine learning is used to do the process automatically. We have also seen before this it is implemented using first derivative of an image or variance of laplacian. Shift from manual process to the automatic signifies growth in this area. In future, this proposed algorithm can be modified and improved using feature extraction algorithm. This concept can also be combined with deep learning neural network where feature extraction step will become automatic and more précised results will be obtained.

\section{REFERENCES}

[1]. R. Dosselmann and X. Yang, "No-Reference Noise and Blur Detection via the Fourier Transform"

[2]. I. Aizenberg, "Type of blur and blur parameters identification using neural network and its application to image restoration", ICANN '02 Proceedings of the International Conference on Artificial Neural Networks, pp. 1231-1236, 2002.

[3]. Ali and Mahmood, "Analysis of Blur Measure Operators for Single Image Blur Segmentation”, Applied Sciences, vol. 8, pp. 1-32, 2018

[4]. L. Guixiong, Z. Xiaoping and Z. Songbin, "Multi-class Classification of Support Vector Machines Based on Double Binary Tree", 2008 Fourth International conference on Natural Computation, October 18-20, 2008, ISBN 978-0-7695-3304-9.

[5]. K. Murat, G. Selami and Y. Sule, "THE IMPORTANCE OF LOGISTIC REGRESSION IMPLEMENTATIONS IN THE TURKISH LIVESTOCK SECTOR AND LOGISTIC REGRESSION IMPLEMENTATIONS/FIELDS”, vol. 16, issue 2, pp. 25-36, 2012.

[6]. J. Thamer and K. Ban, "IMPLEMENTATIONS OF A SIGMOID ACTIVATION FUNCTION FOR NEURAL NETWORK USING FPGA", $13^{\text {th }}$ Scientific Conference of Al- Ma'moon University College, April, 2012.

[7]. Z. Wei and B. Fredrik, "Multi-Scale Blur Estimation and Edge Type Classification for Scene Analysis", International Journal of computer vision, vol. 24, issue 3, pp. 219-250, 1997

[8]. E. James and Z. Steven, "Local Scale control for edge detection and blur estimation", Pattern Analysis and Machine Intelligence, PAMI, vol. 20, issue 7, pp. 699-716,1998.

[9]. F. Rob, S. Barun, H. Aaron, R. Sam and F. William, "Removing camera shake from a single photograph", Association for Computing Machinery, ACM Trans. Graph, vol. 25, issue 3, pp. 787-794, 2006.

[10]. Y. Chen, W. Qiang, Z. Jiajia, "Motion blur detection on lowest directional high-frequency energy," Image Processing (ICIP), 17 $7^{\mathrm{TH}}$ IEEE International Conference on, vol. issue, pp. 2533-2536, September 26-29, 2010.

[11]. K. Vladimir, T. krasimir and B. Ognian, "Blurred image regions detection using wavelet-based histograms and SVM", Intelligent Data Acquisition and Advanced Computing Systems (IDAACS), IEEE $6^{\mathrm{TH}}$ International Conference on, vol. 1, pp. 457-461, September 15-17, 2011. 